\title{
Analysis and solutions of Artifacts on Multi-slice Spiral CT Images
}

\author{
Ziyue Yan ${ }^{1,2}$, Xuelong $\mathrm{Hu}^{2}$, Lifeng Zhang ${ }^{1}$, Seiichi Serikawa ${ }^{1}$ \\ ${ }^{1}$ Department of Electrical Engineering and Electronics, Kyushu Institute of Technology,1-1 Sensui-cho,Tobata-ku, \\ Kitakyushu 804-8550,Japan \\ ${ }^{2}$ School of Information Engineering, Yangzhou University, 198 Huayangxi Road, Hanjiang, Yangzhou 225127, China \\ *Corresponding Author:yanziyue07@gmail.com
}

\begin{abstract}
Comparing the single spiral CT images and multi-slice CT images, analyzing the cause of artifacts that appeared in the CT images, this study aims to contribute to the convenience of the doctor's diagnosis. Through analyzing the patient CT image artifacts, we find the artifacts are produced mainly from two aspects. One comes from equipment itself, then the other one caused by patients themselves. Using the corresponding method, the artifacts could be minimize or be avoid effectively. Especially introduced the solution of metal artifacts, which expect to make a difference in improving the image quality and doctors diagnosis.
\end{abstract}

Keywords: spiral CT, image quality, artifact.

\section{Introduction}

X-ray Computed tomography(CT) has been successfully widely used since the 1970 s, its structure and properties have been perfected and improved. Many generations of CT scanners have been designed to improve their geometrical aspect and consequently to reduce the scanning time. Spiral CT divide into single spiral CT and multislice spiral CT according to the different scanning way.Single spiral CT scanning parameters similar to ordinary CT, X-ray tube voltage is $80 \mathrm{kV}$ to $140 \mathrm{kV}$; X-ray tube current is $50 \mathrm{~mA}$ to $450 \mathrm{~mA}$; the longest scanning time can expose 100 seconds continuously; when scanning, X-ray beam width determined by the collimated width,the thickness is $1 \mathrm{~mm}$ to $10 \mathrm{~mm}$, the moving speed of examining table is $1 \mathrm{~mm} / \mathrm{s}$ to $20 \mathrm{~mm} / \mathrm{s}$, X-ray tube rotated 360 degrees cost for 1 second commonly.At the end of 1998, multislice CT scan technology started in the clinical application which is a new technology in the development of spiral CT. Following the advance of computer technology, CT can be touched maximum of 64 slices scanning.Multi-slice spiral $\mathrm{CT}$ refers to the spiral $\mathrm{CT}$ equipment which installated more rows detector, X-ray tube rotating one week,can complete the multifaceted volume data acquisition and reconstruction of multiple levels of the image.X-ray tube voltage is $120 \mathrm{kV}$ to $140 \mathrm{kV}$; X-ray tube current is $250 \mathrm{~mA}$ to $600 \mathrm{~mA}$; the longest scanning time can expose continuously 125 seconds to 135 seconds.Spiral CT provides a continuous gantry rotation and a continuous table motion as gantry rotates $^{[1]}$.So each view is at different table position and no interscan delay is needed.Comparing with the single spiral CT, multi-slice spiral CT has several advantages,scanning time speed faster,image of spatial resolution more enhancement, perspective of CT positioning more accurate, utilization rate of X-ray more efficient.In spite of these new technologies, artifacts still persist in spiral CT.Artifacts degrade the CT image quality enormously so that the physicians are not able to give a reliable diagnostic because the anatomies are completely distorted or hidden.

\section{Reasons of artifacts in physical structure}

\subsection{Photon starvation artifact}

A photon starvation can lead to the stripy artifact,mainly appear in the near heart, hip and shoulder joint, especially the shoulder joint ${ }^{[2]}$. Artifacts arise because some parts of individual projection can be very noisy due to insufficient photons passing through widest part of patient.In the process of CT image preprocessing, projection noise is inversely proportional to the measured photon, the fewer photons, the more the noise. When these projects are reconstructed by standard algorithm of scanner, the noise is magnified. For photon starvation artifacts have a variety of methods to restrain. Some scanners use current modulation allowing an increase of photon flux (by 
increasing current through the scanner tube) through widest parts without changing the photon flux through narrower parts. In this way, the number of photons received by all detectors will be balanced. But when the harness pass the less attenuation parts, the patient will accept unnecessary $\operatorname{dose}^{[3]}$.

\subsection{Partial volume effect}

In the scanning plane,there are two or more different density of organizational overlapping, CT numbers cannot be accurately measured by any kind of organization in this level, this situation is called a partial volume effect. Due to the effect of partial volume effect,in same level of the different structure of the object which edges contour is oblique line transect, so the outline is not clear. The artifacts produced by the partial volume effect can reduce scanning layer thickness to reduce it.

\subsection{Undersampling artifacts}

According to the shannon law, in order to avoid confusion, the original data acquisition frequency must be equal to or greater than twice the highest frequency of the signal being sampled ${ }^{[2,4]}$. When $\mathrm{CT}$ signal sampling, the $\mathrm{X}$ wiring harness, to a certain direction when the probe radial and angle sampling spacing is bigger than pixel size,this is known as the direction of undersampling.Lack of sampling frames or in a frame have too large sampling interval will produce aliasing strips artifacts. For undersampling artifacts can be handled by adaptive frame synthetic technology.

\section{Patient based artifacts}

\subsection{Motion artifacts}

In the process of CT scanning data collection, if in the fault the analyte have shift, will lead to the projection data is inconsistent so motion artifacts will produced. Normally, when scanning the patient, the patient's moving, physiological movements such as the heart beating, breathing, and gastrointestinal peristalsis and so on make the object enter or leave the scanning plane.Severely injured patients or children frequently move during scanning, causing motion artifacts. Part of artifacts can be avoided, such as the patient's movement and motion artifacts caused by breathing, if patients can cooperate. Some scanners are equipped with the technique of ECG gating which allows synchronizing the data acquisition with the rhythmic beating of the heart. But viscera voluntary movement, such as heart beat and gastrointestinal peristalsis and so on are hard to avoid. For deal with motion artifact can be considered from two aspects : patients and the machine. For example, instruct patients to exhale and inhale; give inject the tranquilizer to patients; make the starting position of the ball tube scanning and movement direction alignment to minimize motion artifacts;some correction algorithms are also proposed for motion artifact removal $^{[5]}$. Crawford et al. developed a pixel-specific filtered back projection algorithm for motion artifact reduction $^{[6]}$. In their algorithm, in-plane motion is corrected by pixel specific reconstruction in the coordinate system associated with the in-plane motion.

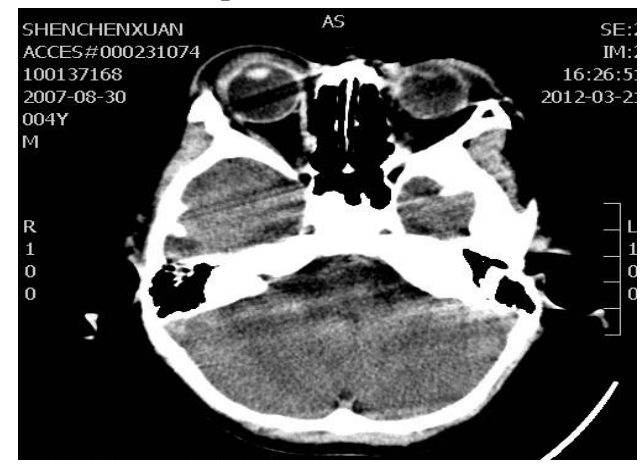

Fig. 1. Example of patient motion artifact

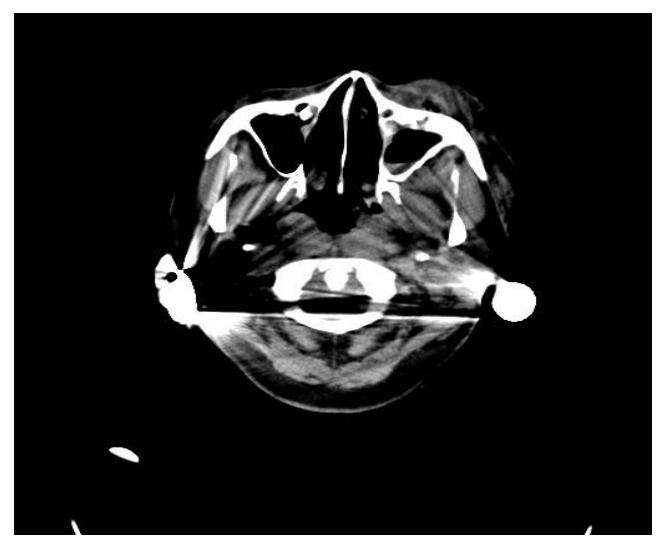

Fig. 2. Example of metal artifact

\subsection{Metal artifacts}

Metal artifacts are caused by the presence of high density objects, such as dental fillings, hip prostheses, metal prosthetic device and so on ${ }^{[7]}$. The artifacts as a streaking effect on an image. The primary reason that streaks occur from metal objects is because the objects exceed the maximum attenuation value that a CT system can image. 
Many older CT number scales assign the number 1000 as the top of the number scale. The number 1000 coincides with the attenuation value of cortical bone, which is primarily the densest structure in the human body. But prosthetic devices or dental fillings which are made of metal have higher attenuation values greater than cortical bone. So, the computer can only assign the highest value it knows. These metallic type objects exceed the dynamic range of the detectors in the detector array. The result is the metallic object damage the image because of streak artifacts.

\section{Solution of Metal artifacts}

\subsection{Metal artifacts}

For metal artifacts can before the scanning, remove the metal objects;to some metal things such as dental fillings, hip prostheses not be able to remove,we can adjust frame angle to avoid metal into the scanning range. Different techniques for metallic artifact reduction have been proposed $^{[8-10]}$.The most efficient methods work on projection matrix. Two different methods have been introduced. In iterative reconstruction methods, the projection data associated with metal objects in projection matrix are disregarded and reconstruction is applied only for non-corrupted data ${ }^{[11-13]}$. Although these algorithms are reliable for incomplete projection data, they must convergence problems and they are computationally expensive for clinical CT scanners deal with convergence problems and they are computationally expensive for clinical CT scanners.

Considering of the basic principle of CT reconstruction images, it wil have two respects of method to removal metal artifact.Firstly from the point of projection compensation, considering the presence of metal object so that the projections are distorted or missed,so before reconstruction will compensate the missing data,the method of projection compensation based on linear interpolation. Secondly,from the point of reconstruction method,the FBP method is not proper again because of the incompleteness of projection, so the iterative reconstruction methods are used here, while the projections of metal are considered missing and are not used in the iterative process. The iterative reconstruction methods include the algebraic reconstruction technique (ART) and the other twos-MLEM and OSEM based on statistical iterative. For projection compensation point of view to the processing of metal area,the first step is the image segmentation to get metal area, projection again in this area. To this range of projection data,interpolation to compensated the projection data.There are three general medical image segmentation methods:based on threshold segmentation method, based on the edg segmentation method based on region segmentation method.

Using the filter back projection algorithm reconstruction of the original image containing metal artifacts; then in the original image segmentation out metal area,in this range on the projection again to get the scope of the metal area in the projection domain; This range of projection data, using linear interpolation method for interpolation to get the projection data after compensated; And use filter back projection algorithm in the projection data use of interpolation to reconstruct, the CT images after corrected insert the metal part image reconstruction image.

\section{Conclusion}

The principle of spiral CT imaging technology is very complex, many factors have a great influence on the image quality. Artifacts degrade the CT image quality and consequently reduce diagnostic quality. Most artifacts can be prevented by using new designs in scanner technology, by careful positioning of patients during scanning, and by optimum selecting of scanner parameters (filter kind, pitch, delivered energy). Some others can be reduced by addressing the problem in software developments. Correctly identify artifacts, understanding the reasons and forms, find out the effective solutions can fully meet the requirements of diagnosis.The quality of CT image has very important practical significance.

\section{References}

(1) Mehran Yazdi and Luc Beaulieu : "Artifacts in Spiral X-ray CT Scanners:Problems and Solutions", Journal of PROCEEDINGS OF WORLD ACADEMY OF SCIENCE,ENGINEERING AND TECHNOLOGY, Vol26, DEC 2007, 376-380, 2007

(2) HE Weihong, Fang Xiangjun,et al : "Analysis and Processing of Artifacts on Helical CT Images”, Journal of Medical Science in Central South China,Vol.139, No.6, pp.692-694, 2011

(3) Barrett JF, Keat N: “ Artifact s in CT: Recognition and avoidance. Radio Graphics Vol.24,No.6, pp.1679-1691, 
(4) Hsieh J: "Image artifacts: appearances, causes and corrections. Computed tomography: principles, design, artifacts and recent advances. Bellingham, Wash: SPIE Press ,pp.167-240,2003

(5) Weng Chuanzheng: "Common causes and solution of CT images artifacts", Journal of Chinese Medical Equipment,Vol.27,No.12,pp.49-51,2006

(6) Qingfei Luo: “ Artifacts in X-ray CT”, Research Imaging Center, University of Texas Health Science Center,

TX 782296C.R.Crawford,K.F.King,C.J.Ritchie, andJ.D.Go dwinRespiratory : "compensation in projection imaging using a magnification and displacement model", IEEE Trans, Vol.15,pp. 327-332, 1996

(7) Robertson DD, Weiss PJ, Fishman EK, et al : "Evaluation of CT techniques for reducing artifacts in the presence of metallic orthopedic implants" , Journal of Comput Assist Tomogr, Vol.12,No.2,pp. 236-241,1988

(8) Ebraheim NA, Coombs R, Rusin JJ,et al: "Reduction of postoperative $\mathrm{CT}$ artifacts of pelvic fractures by use of titanium implants. Orthopedics, Vol.13,pp. 1357-1358, 1990

(9) Ling CC, Schell MC, Working KR, et al:" CT-assisted assessment of bladder and rectum dose in gynecological implants. Int. Journal of Radiat.Oncol. Biol. Phys,Vol.13,pp.1577-1582,1987

(10) Robertson DD, Yuan J, Wang G, et al.: “Total hip prosthesis metal-artifact suppression using iterative deblurring reconstruction “, Journal of Comput. Assist. Tomogr,Vol.21,No.2,pp.293-298,1997

(11) Nuyts J, De Man B, Dupont P,et al: "Iterative reconstruction for helical CT: a simulation study”, Journa of Phys.Med.Biol,Vol.43, No.4, pp.729-737, 1998

(12) Wang G, Vannier MW, Cheng PC, et al.: "Iterative $\mathrm{x}$-ray cone-beam tomography for metal artifact reduction and local region reconstruction" ,Jounral of Microscopy and Microanalysis Vol.5,No.1, pp.58-65, 1999 